\title{
Cell Delivery Systems Using Alginate-Carrageenan Hydrogel Beads and Fibers for Regenerative Medicine Applications
}

\author{
Elena G. Popa, ${ }^{\dagger, \ddagger}$ Manuela E. Gomes, ${ }^{*},+\ddagger$ and Rui L. Reis ${ }^{\dagger, \ddagger}$ \\ ${ }^{\dagger} 3$ B’s Research Group-Biomaterials, Biodegradables and Biomimetics, University of Minho, Headquarters of the European Institute \\ of Excellence on Tissue Engineering and Regenerative Medicine, AvePark, 4806-909 Taipas, Guimarães, Portugal \\ ${ }^{\ddagger}$ ICVS/3B’s—PT Government Associate Laboratory, Braga/Guimarães, Portugal
}

ABSTRACT: The present work was focused on the development and characterization of new hydrogel systems based on natural origin polymers, namely, alginate and carrageenan, into different formats and with adequate properties to sustain the viability of encapsulated cells, envisioning their application as cell delivery vehicles for tissue regeneration. Different formulations of alginate and carrageenan hydrogels and different processing parameters were considered to determine the best conditions required to achieve the most adequate response in terms of the mechanical stability, cell viability, and functionality of the developed systems. The morphology, size, and structure of the hydrogels and their degradation behavior and mechanical properties were evaluated during this study. In addition to cytotoxicity studies, preliminary experiments were carried out to investigate the ability of alginate-carrageenan beads/fibers to encapsulate chondrocytes. The results obtained indicated that the different formulations, both in the form of beads and fibers, have considerable potential as cell-carrier materials for cell delivery in tissue engineering/ regenerative medicine applications.

\section{INTRODUCTION}

Hydrogels are the basis for cell encapsulation/delivery systems, one of the most promising approaches for the delivery of cells and therapeutic agents to the site of interest, allowing a wide range of application in the regenerative medicine field. ${ }^{1}$ During the past decade, several different hydrogels, particularly algal polysaccharides, have been used as cell-delivery matrices. ${ }^{2,3}$ Naturally derived hydrogels have frequently been employed because they are either composed of components or have macromolecular properties similar to the natural extracellular matrix of human tissues. ${ }^{4,5}$ With today's interest in novel renewable polymers, the underexploited marine red algae family belonging to carrageenan seaweeds stimulated our interest as a source of polysaccharides with innovative structure and functional properties. Many different hydrogels have been previously studied for biomedical applications, but carrageenan displays a unique potential in this field and has been very little explored until the present time. Carrageenan is a naturally occurring polysaccharide used far more widely than agar as emulsifier, gelling, thickening and stabilizing agent in pharmaceutical $^{6}$ and industrial formulations. Their relatively low cost promoted their use in environmental and commercial applications as well. ${ }^{7}$ This hydrophilic polysaccharides resemble to some extent the naturally occurring glycosaminoglycans (GAGs) owing to their backbone composition of sulphated disaccharides. ${ }^{8}$ There are three main commercial classes of carrageenan, namely, kappa, which has only one negative charge per disaccharide and produces strong, rigid gels (extracted from Kappaphycus cottonii), the $l$-type, which has intermediate sulfate content and produces soft gels that provide excellent freeze/thaw stability (extracted from Eucheuma spinosum), and $\lambda$, which is highly sulphated, less likely to form a gel structure, but forming gels when mixed with proteins rather than water. ${ }^{9}$ Potassium salts are essential for $\kappa$ - carrageenan hydrogel in order to form this firm gel structure. As the level of potassium is increased, the resulting gel structure becomes tightly aggregated and may cause syneresis (moisture on the gel surface; the separation of liquid from a gel). ${ }^{10} l$ Carrageenan forms elastic, dry gels especially in the presence of calcium salts. The 2-sulfate groups on the outside of the $l$ carrageenan molecule do not allow the helices to aggregate to the same extent as $\kappa$-carrageenan, but form additional bonds through calcium interactions. Because of the ionic nature of carrageenan, the gelling and melting temperatures are dependent almost solely on the concentration of the ions and gelation is strongly influenced by the presence of electrolytes. ${ }^{11}$ This hydrophilic polysaccharide forms a gel with potassium and calcium ions but also shows gelation under salt-free conditions helped by physical bonds being a thermosensitive hydrogel. ${ }^{12}$ Thermoreversible gels, such as carrageenan, melt at elevated temperature and the gelation of the biopolymer is obtained by lowering the temperature. The temperature-induced gelation allows for an easy formation of gels with different shapes, emphasizing the versatility of the carrageenan. Contrary to carrageenan, alginates are probably the most extensively studied and characterized hydrogels for cell encapsulation/delivery. ${ }^{13-15}$ Besides that, alginate has a similar gelation mechanism to carrageenan and, thus, in this study we have focused on the development of hydrogels composed of mixtures of alginate with different types of carrageenan. The mixture of gelled hydrocolloids, in this case, alginate and carrageenan, acts synergistically as a result of the similarity in the type of polysaccharide gelling mechanism and is expected that carrageenan favors the final properties of the mixture, providing

Received: July 12, 2011

Revised: September 30, 2011

Published: October 4, 2011 
Table 1. Conditions Studied Referring to the Alginate-Carrageenan Ratio, Polymeric Concentration, the Salts Used to Crosslink the Gels and Their Concentration, Different Types of Carrageenan $(\kappa$ or $\imath$ ), and Processing Parameters for Obtaining the Hydrogels, Such as Hardening Time, Flow Rate, and Inner Diameters of the Needle

\begin{tabular}{|c|c|c|c|c|c|c|c|}
\hline materials & $\begin{array}{c}\text { alginate- } \\
\text { carrageenan ratio }\end{array}$ & $\begin{array}{c}\text { polymeric } \\
\text { concentration (\%) }\end{array}$ & salt type & $\begin{array}{c}\text { salt concentration } \\
(\%)\end{array}$ & $\begin{array}{l}\text { hardening time } \\
\text { (min) }\end{array}$ & $\begin{array}{l}\text { flow rate (drops/ } \\
\min )\end{array}$ & $\begin{array}{c}\text { inner } \\
\text { diameters }\end{array}$ \\
\hline \multirow{4}{*}{$\begin{array}{l}\text { alginate }-\kappa- \\
\text { carrageenan }\end{array}$} & & 2 & $\mathrm{CaCl}_{2} \cdot \mathrm{KCl}$ & 2 & & & \\
\hline & $5: 5$ & 2.5 & & 2.5 & 15 & 15 & $21 \mathrm{G}$ \\
\hline & $7: 3$ & 3 & & 3 & & & \\
\hline & $8: 2$ & 3.5 & & 3.5 & & & \\
\hline \multirow{4}{*}{$\begin{array}{l}\text { alginate }-l- \\
\text { carrageenan }\end{array}$} & & 2 & $\mathrm{CaCl}_{2} \cdot \mathrm{KCl}$ & 2 & 30 & 30 & $25 \mathrm{G}$ \\
\hline & $5: 5$ & 2.5 & & 2.5 & & & \\
\hline & $7: 3$ & 3 & & 3 & & & \\
\hline & $8: 2$ & 3.5 & & 3.5 & 60 & 60 & $29 \mathrm{G}$ \\
\hline
\end{tabular}

better mechanical properties and tailored degradation profiles. The gelation reaction is produced when cations diffuse into hydrocolloid solution and specific segments of the alginate and carrageenan polymers interact with $\mathrm{K}^{+}$and $\mathrm{Ca}^{2+}$ ions. ${ }^{16}$ In this study, several methodologies were tested to produce a wide range of systems based on carrageenan-alginate mixtures, with different properties in terms of biological and mechanical performance. The processing methodologies used were based on ionotropic gelation and wet spinning to produce different formats like beads and polymeric fibers. ${ }^{17,18}$ The morphology and structure were analyzed by scanning electron microscopy (SEM), the size, shape, and membrane thickness were observed by light microscopy and the mechanical properties of the obtained hydrogels were evaluated by compression tests. Furthermore, the effect of several parameters, such as $\mathrm{pH}$ and different ionic compositions of immersion solution, on the in vitro degradation of the prepared hydrogels was evaluated. The biocompatibility of the developed systems was assessed by standard cytotoxicity assays. Chondrocytes were encapsulated and cultured into the developed beads/fibers and characterized in terms of cell viability (MTS assay), cell proliferation (DNA quantification), and histological staining. In summary, the main goal of this work consisted in the development of an efficient cell delivery system based on combining properties of two natural polymers alginate and carrageenan.

\section{MATERIALS AND METHODS}

Materials. Alginic acid sodium salt from brown algae (71238, Sigma, U.S.A.), $\kappa$-carrageenan (22048, Sigma Fluka, U.S.A.), $l$ carrageenan (22045, Sigma Fluka, U.S.A.), and phosphate buffer saline (P4417-PBS Sigma, U.S.A.) were used without further purification. Calcein-AM (acetoxymethyl ester of calcein-C3099) was purchased from Invitrogen (Portugal).

Development of Alginate-Carrageenan Beads. The hydrogel beads were obtained based on a droplet technique, which consists of the extrusion of the liquid polymer from the tip of a capillary tube continuously until it achieves a critical mass and then detaches and falls into a receiving solution. ${ }^{19}$ Alginate and carrageenan aqueous dispersions were prepared separately by dissolving each of the biopolymers in distilled water heated up at 50 (for alginate) and 60 ${ }^{\circ} \mathrm{C}$ (for carrageenan) under constant stirring from $30 \mathrm{~min}$ to $2 \mathrm{~h}$ until complete dissolution. ${ }^{20}$ The mixtures/blends composed of $8.0 \mathrm{~g}$ alginate solution and $2.0 \mathrm{~g}$ carrageenan solution with concentrations varying from 2 to $3.5 \%$ (blend of alginate with $\kappa$-carrageenan are herein designated by AK and blends of alginate with $l$-carrageenan by AI), were prepared by mixing under constant stirring at $30^{\circ} \mathrm{C}$ for 30 $\mathrm{min}$. Previous to use, all solutions were sterilized by steam power during $30 \mathrm{~min}$ at $120{ }^{\circ} \mathrm{C}$. The solutions were prepared just before use to avoid the initial thermal degradation steps that might affect the biomaterial response. Briefly, the beads were prepared by dropping the mixture using a pump (AL-1000, Alladin Programmable Syringe
Pump) from a $5 \mathrm{~mL}$ disposable syringe equipped with a needle of $25 \mathrm{G}$ inner diameter into the aqueous salt solutions stirred magnetically. The precipitating solution was composed of $100 \mathrm{~mL}$ of $2 \%$ (w/v) $\mathrm{KCl}$ and $\mathrm{CaCl}_{2}$ and the $\mathrm{pH}$ of the solution was previously adjusted to 7.4. After gelation at fixed conditions ( $30 \mathrm{drops} / \mathrm{min}$, agitation rate $50 \mathrm{rpm}$, hardening time $30 \mathrm{~min}, 25 \mathrm{G}$ inner diameter of the needle), the formed beads were separated, washed, and stored at $4{ }^{\circ} \mathrm{C}$ until use. The compositions of the different formulations studied and the different parameters used for their preparation are described in Table 1.

Development of Alginate-Carrageenan Fibers. For the preparation of the polymeric fibers, it was used the same working solutions as described in the previous section for preparing the beads. The fibers were obtained by wet spinning, an easy and reproducible method, which consists in immersing the needle into the cross-linking solution. Each polysaccharide is ionically cross-linked with the corresponding cations, thus, alginate and carrageenan prefer to form a stable gel with $\mathrm{Ca}^{2+}$ and $\mathrm{K}^{+}$, respectively. Alginate-carrageenan fibers were produced using a coagulation bath of $\mathrm{CaCl}_{2} \cdot \mathrm{KCl} 2 \%$, aiming at producing $3 \mathrm{D}$ fiber meshes. Both $\kappa$ - and $l$-carrageenan can shape hydrogels with potassium and calcium salts. They have the ability to form, upon cooling and after addition of the salt, an infinite variety of gels due to development of double helices of polymeric chains into the building blocks of a three-dimensional network. The shape and stability of the hydrogels formed by extrusion techniques are determined by numerous physicochemical factors. Experimental conditions were optimized, such as the distance between the syringe and gelation media, several inner diameters of the needle $(21 \mathrm{G}, 25 \mathrm{G}$, and $29 \mathrm{G}$ ), the flow rate of the polymeric solution falling into gelation bath, the cross-linker concentration, and also the temperature of the system (Table 1).

Physico-Chemical Characterization of the Developed Hydrogels. Morphological Characterization. In this work, the size, shape, and surface characteristics of the developed hydrogels were examined using a stereomicroscope (Zeiss, Stemi 1000 PG-HITEC) and the images were obtained using a camera PowerShot G6, Canon. Scanning electron microscope (SEM - Leica Cambridge S-360, U.K.) was used to observe the surface and the cross section morphology of the hydrogels. For SEM analysis, samples of each condition were washed in PBS, dehydrated in a gradient series of ethanol solutions, and allowed to dry completely at room temperature before sputter coating with gold (Fisons Instruments, Sputter Coater SC502, U.K.).

Degradation Behavior of the Prepared Hydrogels. Degradation studies were carried out by immersion of the hydrogels discs $(\varnothing 7 \pm$ $0.01 \times 10 \pm 0.02 \mathrm{~mm}$ height, $n=3$ ) in phosphate buffered saline (PBS, pH 7.4) and culturing medium (DMEM, $\mathrm{pH} \mathrm{7.4)}$ ) at $37^{\circ} \mathrm{C}$. The hydrogel were lyophilized, weighed (initial weight) before being transferred to $15 \mathrm{~mL}$ Falcon tubes, and soaked in $10 \mathrm{~mL}$ of PBS or DMEM under constant agitation $(60 \mathrm{rpm})$. After each selected degradation time point (final weight), namely, 1, 7, 14, and 21 days, the samples were washed thoroughly with PBS to remove traces of soluble degradation products, salts, or other impurities and then dried until constant weight was achieved. The degradation solutions were changed every 7 days to restore the original level of ions activity. At the end of each degradation period, the dried samples were weighed 
for determination of weight loss. The extent of degradation is commonly determined by calculating the percentage of weight loss (eq $1)$.

$$
\text { weight } \operatorname{loss}(\%)=\frac{\text { initial weight }- \text { final weight }}{\text { initial weight }} \times 100
$$

Mechanical Testing. Uniaxial compression tests were performed to characterize the mechanical behavior of the produced $2.5 \%(\mathrm{w} / \mathrm{v})$ alginate $/ \kappa$-carrageenan hydrogel discs $(\varnothing 15 \pm 0.51 \mathrm{~mm} \times 5.5 \pm 0.46$ $\mathrm{mm}$ height). The hydrogel discs of blends with $l$-carrageenan type were not tested because it was nearly impossible to achieve samples with precise dimensions and thus difficult to obtain results after mechanical tests. The tests were carried out at room temperature conditions using a universal mechanical testing machine (Instron, 4505 Universal Machine). Mechanical testing was performed under compression using a crosshead speed of $5 \mathrm{~mm} / \mathrm{min}$ and the results from tests conducted for at least 10 specimens. The details regarding these methods are presented elsewhere. ${ }^{21}$

Cytotoxicity Evaluation. The cytotoxicity of the constituent's released from the materials was evaluated using cell culture methods, namely MEM extraction test $(72 \mathrm{~h})$ to select the most promising and reject any possible systems that are cytotoxic. In all cytotoxicity tests performed, latex rubber and tissue culture plastic were used as positive and negative controls, respectively. These assays are particularly aimed at establishing the possible toxic effects of leachable released from medical polymers during extraction. The objectives of the MEM extraction test are to evaluate changes in cell morphology and growth inhibition, whereas the MTS (3-(4,5-dimethylthiazol-2-yl)-5(3-carboxymethoxyphenyl)-2(4-sulfofenyl)- $2 \mathrm{H}$-tetrazolium) assay determines whether cells are metabolically active. ${ }^{22}$ The detailed method is presented elsewhere. ${ }^{23}$ Briefly, for biocompatibility assessment of the different formulations of the developed hydrogels a mouse fibroblastlike cell line, L929 (ECACC- European Collection of Cell Cultures, U.K.) was used. Cells were fed with Dulbecco's modified Eagle's medium (DMEM, Sigma-Aldrich), supplemented with $10 \%$ fetal bovine serum (FBS, Gibco, U.K.) and $1 \%$ antibiotic/antimycotic (A/B, Gibco, U.K.) solution. L929 cells were incubated at $37{ }^{\circ} \mathrm{C}$ in an atmosphere containing $5 \% \mathrm{CO}_{2}$ until $90 \%$ confluence was achieved. Then, a cell suspension with a concentration of $2 \times 10^{4}$ cells $/ \mathrm{mL}$ (4000 cells/well) was prepared and seeded onto 96-well plates. Extract of the developed materials were prepared by incubating the hydrogels with media containing serum at an extraction ratio of $3 \mathrm{~cm}^{2} / \mathrm{ml}$ for 24 $\mathrm{h}$ at $37{ }^{\circ} \mathrm{C}$. One hundred microliter of the extracts of the hydrogel, negative control (standard culture medium), and positive control (latex rubber) were placed on $80 \%$ confluent monolayer of cells. The absorbance of the resulting solution in each well was recorded immediately at $490 \mathrm{~nm}$ using an automated Multi-Mode Microplate Reader (Synergy HT, Bio-Tek Instruments, U.S.A.). Results were presented as OD (optical density) after extraction of the blank value (i.e., medium only) and we conducted all cytotoxicity screening tests by using six replicates. The results were expressed as percentage of cell viability calculated using the following equation:

$$
\text { cell viability }(\%)=\frac{\text { OD sample }}{\text { OD control }} \times 100
$$

where OD sample is the optical density obtained in the cells exposed to each extract and OD control is the OD obtained in the cells incubated with the culture medium only (negative control).

Encapsulation of ATDC5 Cells into Alginate/Carrageenan Hydrogels. Cell encapsulation experiments were performed to assess the viability of cells incorporated in the developed hydrogels and to further select/optimize the developed formulations as well as the encapsulation conditions. ATDC5 cells, a cell line established from chondrocytic cells of a mouse embryonal carcinoma (mouse 129 teratocarcinoma AT805 derived, ECACC, U.K.) was selected for this work, because these cells are more biologically active in a $3 \mathrm{D}$ environment and have been extensively studied to assess their role in producing, maintaining, and remodeling the cartilage ECM. ${ }^{24}$ As previous experiments revealed that the shape of the hydrogel did not affect differently the viability or proliferation of the cells, herein it was decided to present results based on hydrogels with fiber shape. Prior to cell culture studies, alginate/carrageenan solutions were sterilized by heat for $30 \mathrm{~min}$ at $120{ }^{\circ} \mathrm{C}$. The cells were expanded in cell culture medium (Ham's F-12 medium (DMEM-F12, Gibco, U.K.) supplemented with 10\% FBS (Gibco, U.K.), 2 mM L-glutamine (Sigma), and $1 \% \mathrm{~A} / \mathrm{B}$ antibiotic solution (Gibco, U.K.) at $37{ }^{\circ} \mathrm{C}$ in $\mathrm{CO}_{2}$ incubator until obtaining the necessary number of cells for the experiments. After trypsinization and centrifugation, the cell suspension obtained was diluted to encapsulate $1 \times 10^{6} \mathrm{cell} / \mathrm{mL}$ of the polymeric solution to produce the beads/fibers, as previously described. The beads/fibers with encapsulated cells were then placed into nonadherent 24-well plates and maintained in culture for several periods of time $(1,7,14$, and 21 days) at $37{ }^{\circ} \mathrm{C}$ in a $\mathrm{CO}_{2}$ incubator. At the end of each time of culture, the beads/fibers with the encapsulated cells were retrieved, washed with PBS solution, and maintained at $4{ }^{\circ} \mathrm{C}$ in PBS for further characterization studies.

Light Optical Microscopy and Fluorescence Staining with Calcein-AM. ATDC5 cell morphology after encapsulation in beads and fibers was observed under inverted light microscope (Zeiss, Axiovert 40 PG-HITEC). The viability of ATDC5 cell line was investigated using Calcein AM fluorescence labeling after 21 days in culture. For this assay, the samples were washed with PBS, placed in a new well plate with $1 \mathrm{~mL}$ of DMEM and $2 \mu \mathrm{L}$ of a calcein-AM solution (at $1 / 1000$ dilution), and incubated for $15-30 \mathrm{~min}$ at $37{ }^{\circ} \mathrm{C}$ in $5 \%$ $\mathrm{CO}_{2}$. The fluorescence staining was washed and replaced by $500 \mu \mathrm{L}$ of formalin $(10 \%)$ for $10 \mathrm{~min}$ at room temperature to fix the cells. Afterward, the formalin was removed, and the beads and fibers were rinsed twice in PBS and observed under a reflected/transmitted light microscope (Zeiss, Axiocam MRc5).

Assessing the Metabolic Activity (MTS Assay). The alginatecarrageenan polymers with encapsulated ATDC5 cells were cultured for $1,7,14$, and 21 days; cell-free hydrogel samples were kept in the same conditions to be used as experimental controls. After each of the selected time points of culture, the medium was removed; samples were washed with PBS and assessed for their metabolic activity using the CellTiter 96 Aqueous One Solution Cell Proliferation Assay (MTS, Promega). Briefly, cell-hydrogel constructs $(n=3)$ were washed in PBS and placed in a mixture containing serum-free cell culture medium DMEM (without phenol red) and MTS reagent at a 5:1 ratio and incubated for $3 \mathrm{~h}$ at $37^{\circ} \mathrm{C}$ in a humidified atmosphere containing $5 \% \mathrm{CO}_{2}$ at the end of which $100 \mathrm{~mL}(n=6)$ was transferred to 96-well plates and the OD determined at $490 \mathrm{~nm}$.

Cell Proliferation by DNA Quantification. ATDC5 cell proliferation in the hydrogels was determined using a fluorimetric double-strand DNA quantification kit (Quant-iT PicoGreen dsDNA reagent, Molecular Probes, Invitrogen). For this purpose, samples that were collected at $1,7,14$, and 21 days of culturing were transferred into $1.5 \mathrm{~mL}$ microtubes containing $1 \mathrm{~mL}$ of ultrapure water. ATDC5 cell-hydrogel constructs and the hydrogel alone (without cells), used as control, were incubated for $1 \mathrm{~h}$ at $37^{\circ} \mathrm{C}$ in a water bath, and were then stored in a $-80^{\circ} \mathrm{C}$ freezer until they were tested. Prior to dsDNA quantification, constructs were thawed and sonicated for $15 \mathrm{~min}$. Samples and standards (ranging from 0 to $2 \mu \mathrm{g} / \mathrm{mL}$ ) were prepared and mixed with a PicoGreen solution in a 200:1 ratio and were placed on opaque 96-well plate. Each sample or standard was made in triplicate. The plate was incubated for $10 \mathrm{~min}$ in the dark, and fluorescence was measured on a microplate reader (Synergy HT, BioTek Instruments) with an excitation of $485 / 20 \mathrm{~nm}$ and an emission of $528 / 20 \mathrm{~nm}$. A standard curve was created, and sample DNA values were read from the standard graph.

Histological Analysis (H\&E Staining). Hydrogel fiber samples were collected at each time point of the study and previously fixed with $10 \%$ formalin were processed by a series of dehydration steps, embedded in paraffin, and sectioned at $4 \mu \mathrm{m}$ (HM355S Rotary Microtome, Thermo Scientific). Haematoxylin and eosin (H\&E) staining was conducted in automatic staining equipment (Microm HMS 740) and then cleared in xylene (VWR) and mounted for further analysis. 


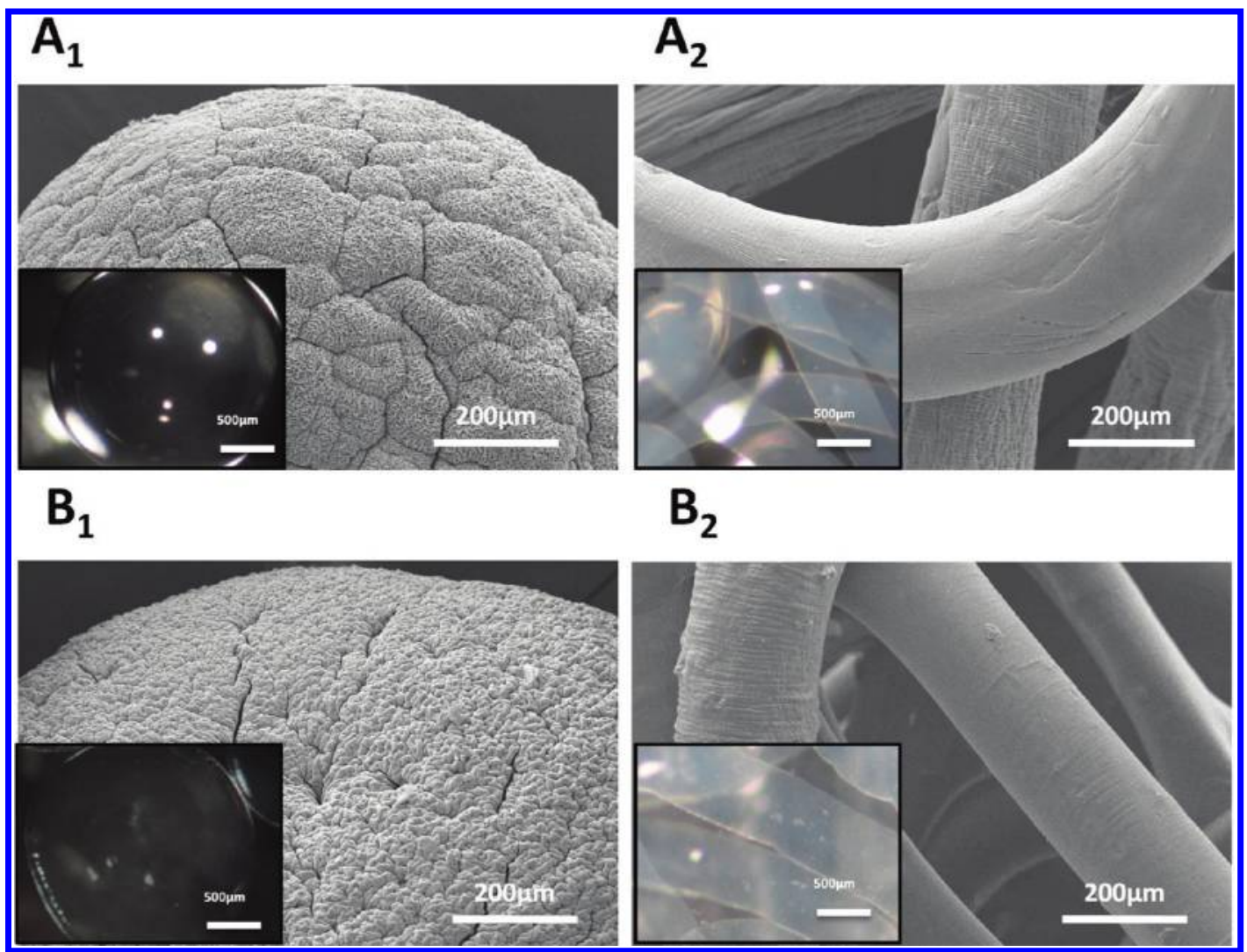

Figure 1. SEM micrographs of alginate-carrageenan blend surface (8:2) of beads $A_{1}, A K 2.5 \%, B_{1}, A I 3.5 \%$, and fibers $A_{2}, A K 2.5 \%, B_{2}, A I 3.5 \%$; embedded images correspond to light microscopy pictures of the same samples in wet state, precipitated in $2 \%(\mathrm{w} / \mathrm{v}) \mathrm{CaCl}{ }_{2} \cdot \mathrm{KCl}$ and hardening time of $30 \mathrm{~min}$.

Statistic Analysis. Data of compression, degradation, MTS, and DNA assay are presented as means \pm standard deviations, with $n=6$ for each group. For statistic analysis multiple pair's comparison has been performed using One-Way ANOVA, Tukey method with a significance level of 0.05 , using OriginPro 8 program. First an F-test was used to ascertain about the data normality of the results, namely, to determine the equality of variance.

\section{RESULTS}

Development of Carrageenan-Alginate Hydrogel Beads and Fibers. This study has focused on the development of novel hydrogels based on natural-origin polymers, namely, alginate and carrageenan. To obtain the optimum hydrogel formulation, several aspects were considered, such as, the use of different types of carrageenan, $l$ and $\kappa$, different concentrations of the polymers, and various concentrations of the salts were evaluated (Table 1). Parameters related to the processing method, such as flow rate and needle diameter, show a direct influence in the size of the produced beads; increasing the flow rate of the polymeric solution resulted in larger bead diameter and using needles with small diameter resulted in smaller droplets. The concentration and molecular structure of carrageenan and alginate, as well as the concentrations of calcium and potassium chloride, have an important effect in the gelation process. Other aspects related to the ionotropic gelation method by droplet formation, namely, droplet rates, flow rate of the viscous solution, hardening time, needle distance, needle diameter, and $\mathrm{pH}$ of the solution were taken into account for the optimization of the gel formation. Contrary to $\kappa$-carrageenan, no spherical beads were formed with formulations based on $l$-carrageenan using the selected salt solutions (Table 1). The optimum concentration of the reactants was determined using empirically classical methods. Experiments with $\kappa$-carrageenan type showed that the best bead formation were obtained by dropping $2.5 \%$ $\kappa$-carrageenan polymer into $5 \%$ potassium chloride solution while for $l$-carrageenan type the best spheres were obtained with $3.5 \%$ dispersion and $2 \%$ calcium chloride. Higher concentrations of the polymer dispersion used in this study could not be dropped so easily from the needle due to the high viscosity. The same difficulty was observed with lower concentrations, which led to unstable gels in terms of shape and structure. Alginate/ $\kappa$-carrageenan hydrogel blends were formed faster than $\kappa$-carrageenan alone, due to the quick and strong reaction of alginate with $\mathrm{Ca}^{2+}$ ions. In addition, carrageenan basically formed softer beads with less spherical shape than the alginate-based beads. Alginate/carrageenan fibers produced by immersion of the polymeric solutions inside the chemical bath containing potassium and calcium ions presented the same gelling properties as in the case of beads formation.

Morphologic Characteristics of Alginate/Carrageenan Hydrogels. SEM micrographs of the hydrogels, namely, AK $2.5 \%$ (Figure $1, \mathrm{~A}_{1}$, beads, and $\mathrm{A}_{2}$, fibers) and $\mathrm{AI} 3.5 \%$ (Figure $1, B_{1}$, beads, and $B_{2}$, fibers) showed no significant differences regarding the surface morphology. Nevertheless, blends with $\kappa$ carrageenan $2.5 \%$ concentration and blends with $3.5 \%$ concentration for the $l$-carrageenan type led to the formation of the most stable and well-defined shapes. From the light microscopy images of the beads (Figure 1, $\mathrm{A}_{1}, \mathrm{AK} 2.5 \%, \mathrm{~B}_{1}, \mathrm{AI}$ $3.5 \%$ ) and fibers (Figure 1, $\mathrm{A}_{2}, \mathrm{AK} 2.5 \%, \mathrm{~B}_{2}, \mathrm{AI} 3.5 \%$ ) in wet 


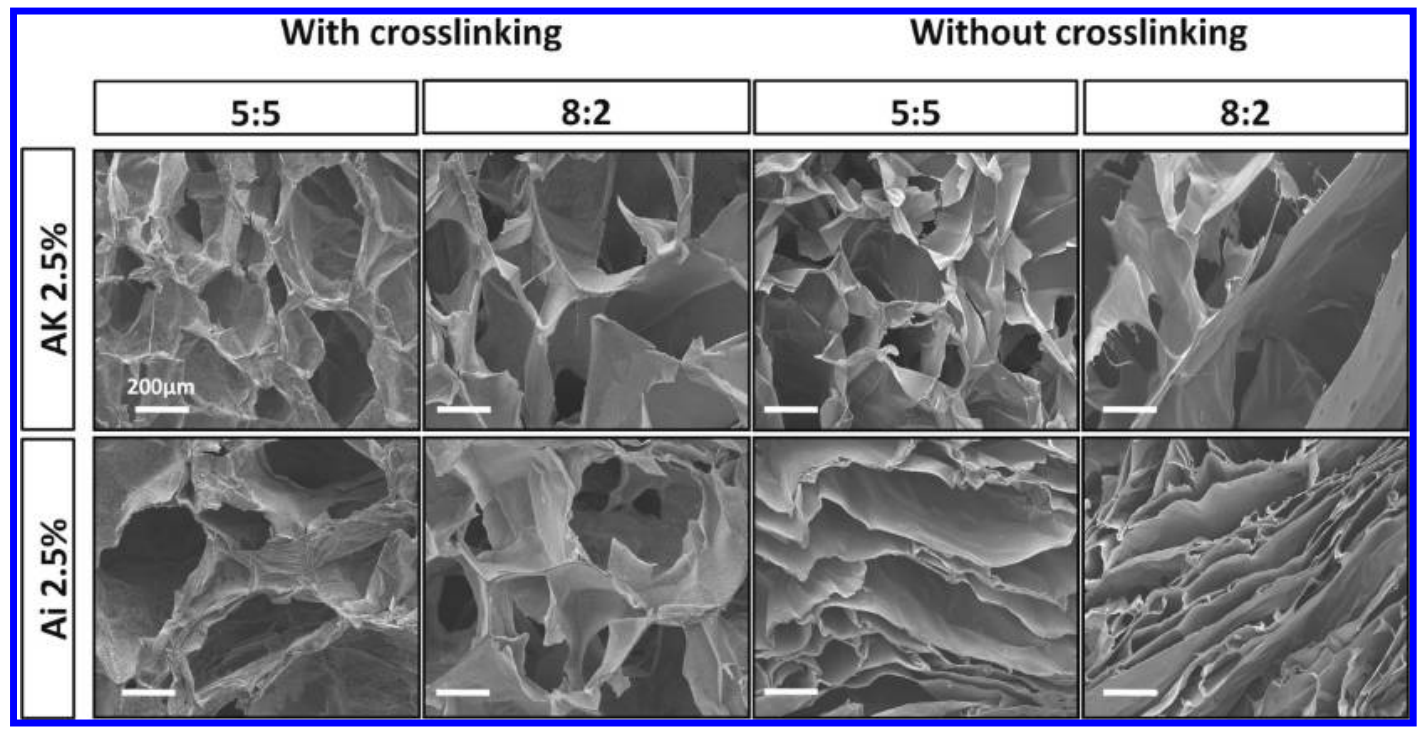

Figure 2. Scanning electron microscopy images of freeze-dried cross sections presenting the morphology and porosity of AK $2.5 \%$ and AI $2.5 \%$ hydrogel blends (5:5 and 8:2 polymeric ratio, with cross-linking agent and without cross-linking, $\mathrm{CaCl}_{2} \cdot \mathrm{KCl}$ ) at $100 \times$ magnifications and $200 \mu \mathrm{m}$.
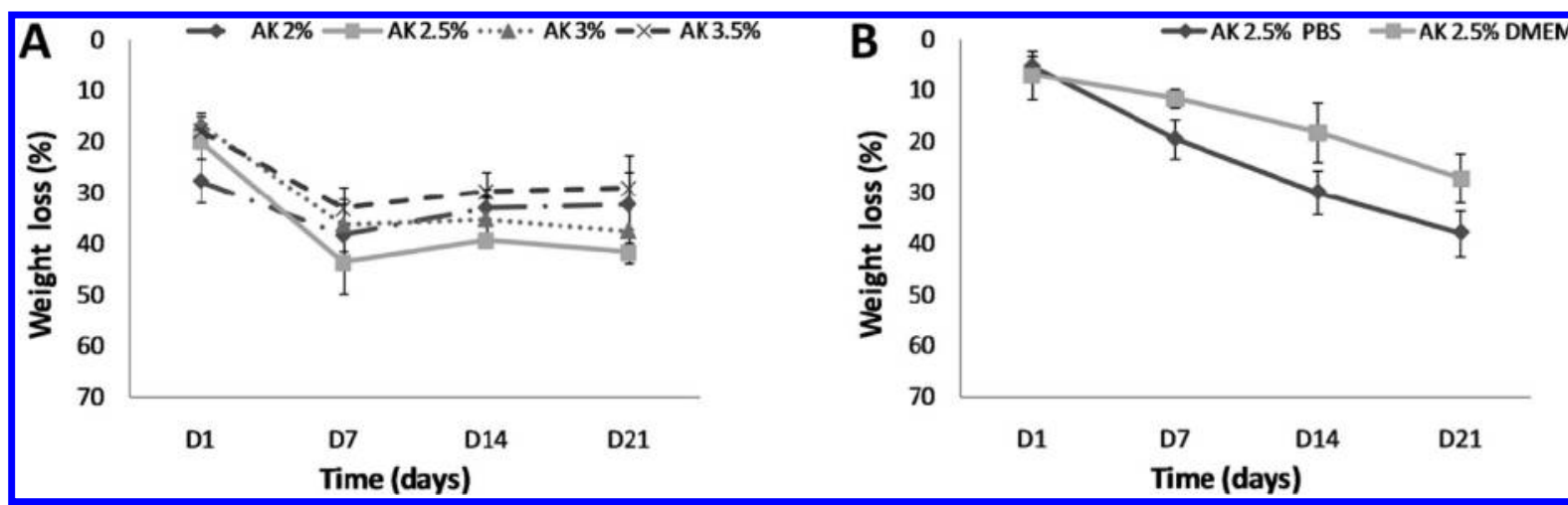

Figure 3. Representation of weight loss percentage vs time of alginate/ $\kappa$-carrageenan discs with different polymeric concentrations immersed in culture medium (DMEM) (A) and measurements after immersion in PBS and DMEM solutions at $37{ }^{\circ} \mathrm{C}$ for AK $2.5 \%$ hydrogel blend (B). Values reported correspond to averages $(n=3) \pm$ standard deviation.

state, one can observe the smooth and homogeneous surface and the well-defined and stable shapes. The mean beads dimension for the AK $2.5 \%$ (8:2) formulation using $21 \mathrm{G}$ needle was $2.76 \pm 0.14 \mathrm{~mm}$, using the $25 \mathrm{G}$ diameter needle the mean dimensions was $2.16 \pm 0.13 \mathrm{~mm}$ and when applying the needle of $29 \mathrm{G}$ was $1.08 \pm 0.07 \mathrm{~mm}$. The mean fiber dimensions for the AK $2.5 \%(8: 2)$ formulation were typically lower and ranging from $1.1 \pm 0.08(21 \mathrm{G}), 0.9 \pm 0.08(25 \mathrm{G})$, and $0.56 \pm$ $0.08 \mathrm{~mm}(29 \mathrm{G})$. Various complexes of hydrogels were prepared from different (5:5 and 8:2) alginate/carrageenan ratios and variations of carrageenan type, namely, kappa and iota, were assessed (Figure 2). The hydrogels were cross-linked in reaction with $\mathrm{CaCl}_{2}$ and $\mathrm{KCl}$ and SEM (Scanning Electron Microscopy) images of cross-linked and noncross-linked hydrogels were compared. These images show that the pore size was homogenously distributed and increased with increasing carrageenan content. More than $60 \%$ of the hydrogel volume or greater, presented porosity, indicating that the carrageenan content is a key factor for controlling the pore size. Two different pore morphologies are obvious in the non-crosslinked, mainly due to the type of carrageenan used, kappa hydrogels exhibiting round porosity, small and circular cavities, and iota presenting longitudinal pores and large and elongated cavities. Regardless of the hydrogel composition, the pore size (ranging from 100-200 $\mu \mathrm{m}$ ) of cross-linked hydrogels was smaller than the pore size of non-cross-linked ones and many pores in cross-linked hydrogels were deformed by cross-linking (Figure 2). The cross-linked hydrogel prepared from an 8:2 alginate/carrageenan mixture using $\kappa$-type, followed by reaction with $\mathrm{CaCl}_{2} \cdot \mathrm{KCl}$, enabled the formation of an uniform hydrogel provided by a good miscibility and, thus, was found to be the most suitable hydrogels formulation.

In Vitro Degradation Studies. The aim of the degradation studies was to evaluate the behavior of the developed materials in simulated physiological conditions. Therefore, the degradation of the hydrogels was monitored as a function of incubation time in PBS and DMEM at $37{ }^{\circ} \mathrm{C}$, as shown in Figure 3. During the degradation time, most of the formulations based on $l$-carrageenan with a low concentration showed to be very unstable, disintegrating after a few days in the degradation media, and therefore, only the kappa formulation was further analyzed. Moreover, it was found that the hydrogels obtained with higher polymeric concentration and a polymeric ratio of $8: 2$ had acceptable stability, and therefore, these were selected for further and detailed characterization. The polymeric concentration of the hydrogel blend had a significant influence on the weight loss. In the first days of the experiment, the hydrogel blends with lower 
concentrations presented the highest weight loss rate. The hydrogels with 2 and $2.5 \%$ polymer concentrations lost about $35-45 \%$ of their weight in the first 7 days of the experiment (Figure 3A). The blended hydrogels with higher concentration (3 and 3.5\%) showed a lower weight loss rate. At day 21, the AK $2.5 \%$ formulation presents the highest weight loss. Therefore, this formulation was selected for further characterization of the degradation behavior and, thus, immersed in PBS and DMEM medium for up to 21 days. The results obtained from these experiments are presented in Figure 3B. Weight loss results showed fast degradation behavior in PBS solution as compared to DMEM medium.

Mechanical Characterization by Compression Tests. The mechanical properties of the blend consisting of alginate and $\kappa$-carrageenan $2.5 \%(\mathrm{w} / \mathrm{v}$, ratio $8: 2)$ were examined in compression experiments. The Young's modulus for AK $2.5 \%$ $(8: 2)$ in compression was found to be $0.7 \pm 0.25 \mathrm{MPa}$.

Biological Studies: Cytotoxicity Assays. The cytotoxicity of the components that leach out of the developed hydrogels was evaluated by a viability assay (the MTS test). Table 2 shows the results of the cellular metabolic activity

Table 2. Results Obtained from Cytotoxicity Tests Performed Using Extracts of AK and AI Hydrogels with Different Concentrations ${ }^{a}$

\begin{tabular}{lllc} 
samples $^{b}$ & \multicolumn{1}{c}{ day 1} & \multicolumn{1}{c}{ day 3} & day 7 \\
AK2.5\% & $74.13 \pm 0.72$ & $40.57 \pm 2.68$ & $54.39 \pm 0.78$ \\
AK3\% & $128.26 \pm 6.89$ & $123.00 \pm 1.73$ & $98.77 \pm 4.88$ \\
AK3.5\% & $106.89 \pm 0.62$ & $96.23 \pm 3.22$ & $90.26 \pm 5.40$ \\
AI2.5\% & $55.99 \pm 5.39$ & $28.59 \pm 2.03$ & $35.89 \pm 0.31$ \\
AI3\% & $91.26 \pm 5.50$ & $86.56 \pm 2.16$ & $75.24 \pm 0.89$ \\
AI3.5\% & $106.89 \pm 0.62$ & $96.23 \pm 3.22$ & $90.26 \pm 5.40$
\end{tabular}

${ }^{a_{\text {The }}}$ results are based on the MTS test performed after 1,3 , and 7 days of culture. ${ }^{b}$ We determined the values by taking into account the fact that TCPs (negative control) corresponded to $100 \%$. Latex was used as a positive control of cell death and produced cell viability values that were considered to be negligible.

obtained using extracts of the materials under study. The results obtained revealed that the extracts from the samples with a higher polymeric concentration did not affect the viability of the cell line L929. This test demonstrates the extremely low cytotoxicity levels of the alginate-carrageenan blend when using higher concentrations of the polymers. On the other hand, the hydrogels with the lower polymeric concentrations presented a higher cytotoxicity.

Optical Microscopy and Fluorescence Staining with Calcein-AM. The cells were encapsulated within the $2.5 \%(\mathrm{w} /$ v) alginate $/ \kappa$-carrageenan hydrogel blend in beads and fibers. The light microscopic images presenting ATDC5 encapsulated in beads and fibers show a uniform cell distribution after 21 days in culture, a homogeneous cell density, and smooth, well delimited shape of the hydrogels. Calcein-AM fluorescence staining was conducted to assess the ATDC5 cells viability and distribution within the hydrogels. The majority of the cells were positive for calcein-AM, which indicates a dominance of viable cells over a period of 21 days in culture. Three weeks postencapsulation, ATDC5 chondrocytes cells exhibited round shapes, uniform cell distribution into the hydrogel, and a high cellular density (Figure 4). These results show that both shape conditions of the hydrogels blend are not cytotoxic and that the temperature cycle used to promote the sol-gel transition does not affect cell viability.

Viability and Proliferation of Cells Encapsulated in the Developed Hydrogels. In this study, the influence of two parameters on the viability and cell proliferation were analyzed, namely, the ratio of the two types of carrageenan, kappa and iota, and also the concentration of the cross-linker used for the formation of the 3D construct. Generally, from the MTS and DNA results, it was found that the ATDC5 cells remain viable and proliferate for up to 21 days of encapsulation/culture in the $3 \mathrm{D}$ hydrogels developed. From the MTS assay results (Figure 5A), we can observe that the formulations based on $\kappa$-carrageenan enabled higher cell viability as compared to those based on $l$-carrageenan. This difference in terms of cellular response could be related to the chemical composition of $l$-carrageenan, which presents more sulphated groups than $\kappa$-carrageenan and, thus, the lower strength of its network, ${ }^{25}$ enables an easier leach out of cells from the tested samples. Analyzing the importance of the polymers ratio used, we can notice an increase in the metabolic activity obtained for the ATDC5 cells encapsulated in the formulation with higher alginate content, corresponding to the ratio 8:2, but not significantly different. For further biological characterization, it was selected the blend with $2.5 \%(\mathrm{w} / \mathrm{v})$ alginate and $\kappa$-carrageenan with a ratio of $8: 2$. The influence of several concentrations of the reticulation agent $\left(\mathrm{CaCl}_{2} \cdot \mathrm{KCl}\right)$ and their biological response were investigated (Figure 5B,C). The results obtained from the MTS assay showed that a lower concentration of the reticulation agent has a positive effect in the cellular response especially for $\mathrm{AK} 2.5 \%$ formulation. Moreover, we can observe a higher cell proliferation with the formulation exposed to lower concentration of $\mathrm{CaCl}_{2} \cdot \mathrm{KCl}$ precipitation bath complementary to the MTS assay results (Figure 5C)

Histological Analysis (H\&E). The morphology of the cells residing in the hydrogel blends containing one of the two types of carrageenan and with different polymeric concentration was further observed by $\mathrm{H} \& \mathrm{E}$ staining. Histological analysis of samples taken after 21 days of culture was performed using hematoxylin-eosin ( $\mathrm{H} \& \mathrm{E})$ for regular morphological cellular analysis (Figure 6). In a typical tissue, nuclei are stained blue, whereas the cytoplasm and extracellular matrix have varying degrees of pink staining. From the histological results we observed that the cells are viable, presenting homogeneous distribution and exhibit a clearly delimited nucleus over the culturing period. Encapsulated ATDC5 cells exhibit a normal morphology, some presenting small nuclei mostly in the initial culturing period, others large proliferating nuclei by 21 days of culture and in some cases, one can notice cell clustered formation in general in kappa condition. Additionally, after encapsulating ATDC5 chondrocytic cells, we observed that they were able to proliferate and maintain their typical chondrocytes morphology, namely, the lacunae aspect.

\section{DISCUSSION}

Hydrogels can be used for cell delivery, ${ }^{26}$ as well as growth factor or drug delivery. ${ }^{27}$ Hydrogels derived from naturally occurring polysaccharides mimic many features of extracellular matrix (ECM) and thus have the potential to direct the migration, growth and organization of encapsulated and transplanted cells during tissue regeneration. ${ }^{28}$ In this study, a new forming biodegradable hydrogel system was developed, which may be used as a carrier for cell delivery purpose. 


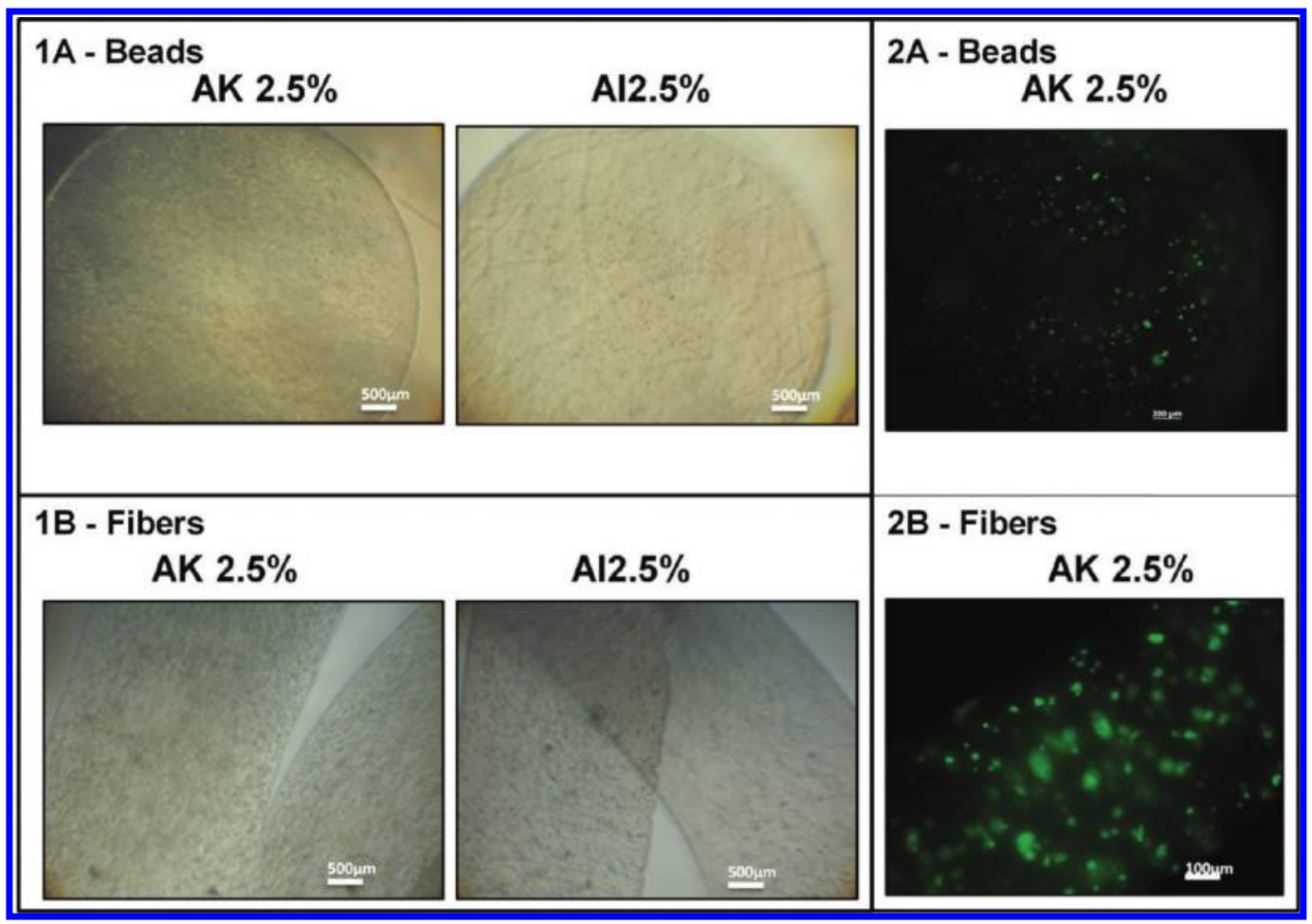

Figure 4. Inverted light microscopic images of ATDC5 cell encapsulated in beads and fibers (1A and 1B) and fluorescence staining, calcein-AM (green, 2A and 2B) images depicting viable cells stained at day 21 of culture.

Alginate hydrogels are fast gelation systems, hard to control, often the resulting structure is not uniform and mechanically strong and thus is difficult to achieve complex-shaped 3-D structures. ${ }^{29}$ We expected that adding carrageenan hydrogel to the system could overcome some of these disadvantages. The hydrogels were obtained by the cross-linking of alginate and $\kappa$ carrageenan with $\mathrm{Ca}^{2+}, \mathrm{K}^{+}$ions at a stable physiological $\mathrm{pH}$. Different volume ratios of the hydrogel blend were prepared for assessing the best formulation in terms of degradation profile, mechanical stability, and cell behavior. It was possible to process alginate-carrageenan in different ways, enabling to obtain various structures/shapes, such as beads and fibers, which can be used in different approaches/strategies for the delivery of cells. The smooth appearance of the hydrogels is clearly observed from the microscopic images of the beads and fibers in dry and wet state (Figure 1). This demonstrates that the rigid, glassy alginate polymer can be blended with the highly flexible, amorphous $\kappa$-carrageenan polymer, creating smooth morphologies and enhance the thermal stability. ${ }^{20,30}$ The images obtained from the cross sections of the hydrogels suggest that the morphology of the blends depends on the type of carrageenan used, and this may result from the fact that $\kappa$ carrageenan gives harder gels than $l$-carrageenan with $\mathrm{CaCl}_{2}$ (The United States Pharmacopeia XXIII, 1995). The SEM images of the freeze-dried hydrogels also demonstrated that a higher content of $\kappa$-carrageenan results in the formation of smaller, more round, pores and tighter network structure in the blend hydrogels (Figure 2). A similar behavior was register in blends made with carrageenan and gelatin where the average pore size and porosity decreased with increasing $\kappa$-carrageenan content as well, owing to the high molecular weight and viscosity of the $\kappa$-carrageenan solution. ${ }^{31}$ Moreover, the morphology and porosity is highly affected by the gelling agents, as compared to the degree of cross-linking with ions.
However, our results showed that the type of carrageenan used in the blend composition and the alginate/carrageenan ratio are both important parameters for pore size control and shape of the hydrogel. ${ }^{32}$ To promote the regeneration of tissues, it is important that the proposed hydrogels can delivery cells at the target site but also maintain its structure under physiological conditions, similar to those found in vivo, for enough time so that they can perform a protective role for the implanted cells and thus ensure their functionality. ${ }^{33}$ In engineered hydrogel for cell encapsulation/delivery degradation can alter the diffusion of nutrients, waste, and cell-material interactions. The performed degradation studies allowed characterizing the developed hydrogels in simulated physiological conditions like PBS and DMEM immersion mediums at $37{ }^{\circ} \mathrm{C}$ by mimicking the behavior in the in vivo scenario. As mentioned before, it was found significant differences and systematic variation in the formation and stability of the hydrogel, depending on the polysaccharide used, with $\kappa$-carrageenan being firm and rigid and $l$-carrageenan being soft and elastic. Having in mind these physicochemical differences between $\kappa$ - and $l$-carrageenan type on the blend formation, only the $\kappa$-carrageenan polymer has been taken into account for degradation characterization. The hydrogel blend with low concentration revealed less stability, consequently increasing the exposure of polymer chains to water molecules and significantly leading to water absorption enhancement and faster weight loss (Figure 3). Also, the carrageenan component of blend would be expected to have a greater preference for water sorption than alginate because of the presence of highly polar sulfate groups in the structure. ${ }^{30}$ Polymeric concentration influences many properties of the resulting hydrogels. In general, an increase in the polymer concentration results in a decrease in water content and mass weight loss. In other words, the hydrogels with lower concentrations present a faster degradation rate, losing almost 


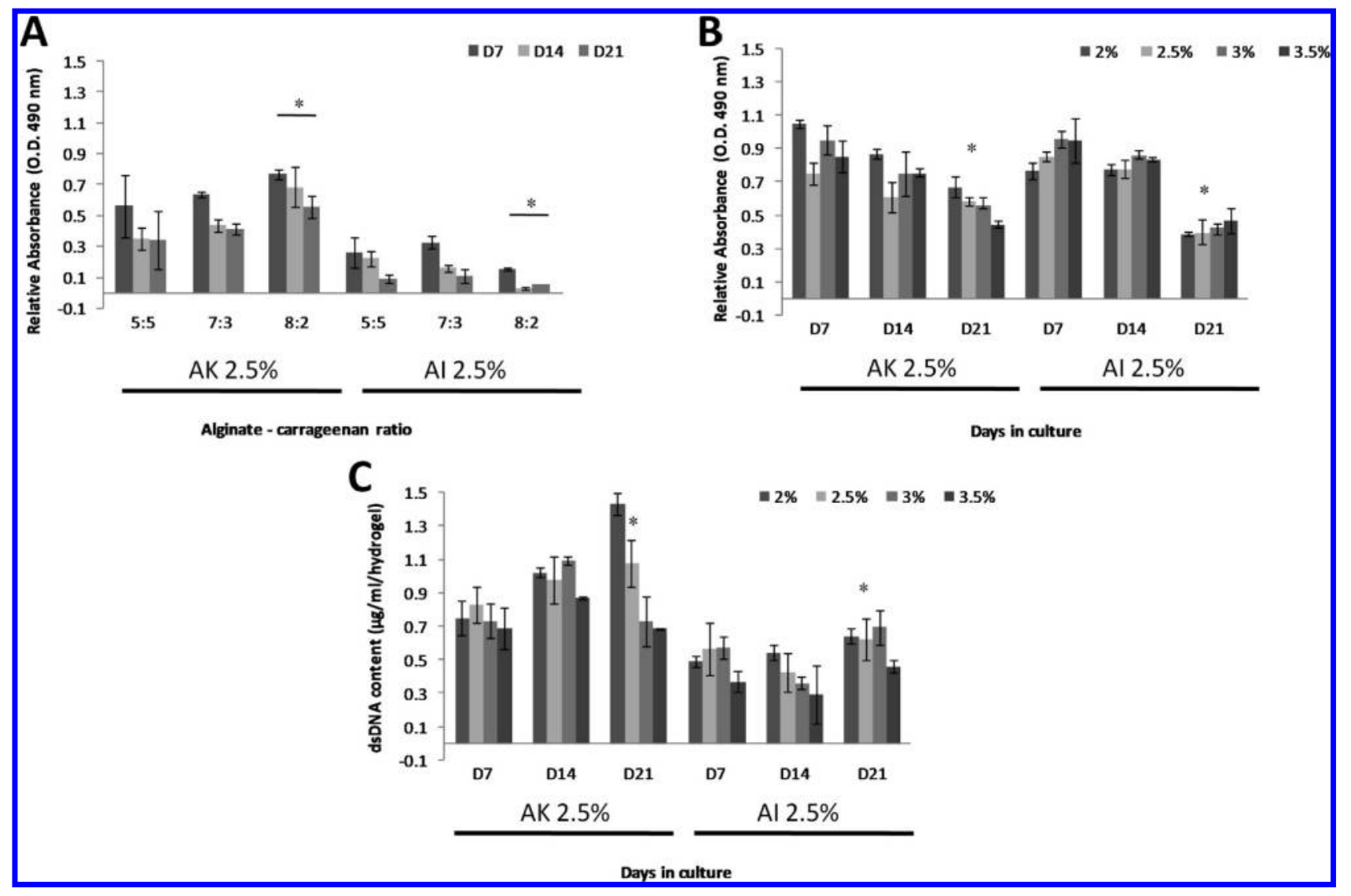

Figure 5. (A) Metabolic activity of ATDC5 cells encapsulated in the $2.5 \%$ alginate-carrageenan with a different polymeric ratio. (B) Hydrogels exposed to different concentration of the $\mathrm{CaCl}_{2} \cdot \mathrm{KCl}$ coagulation bath. The results are based on the MTS test performed after 1, 7, 14, and 21 days of culture. (C) Cell proliferation of ATDC5 cells entrapped in hydrogels blend using the two types of carrageenan with different concentration of the precipitation bath $\left(\mathrm{CaCl}_{2} \cdot \mathrm{KCl}\right)$. The results are based on the DNA quantification test performed at 7,14 , and 21 days of culture. The symbols $*$ indicates statistical significance of the formulation AK 2.5\% and AI 2.5\% (ratio 8:2).

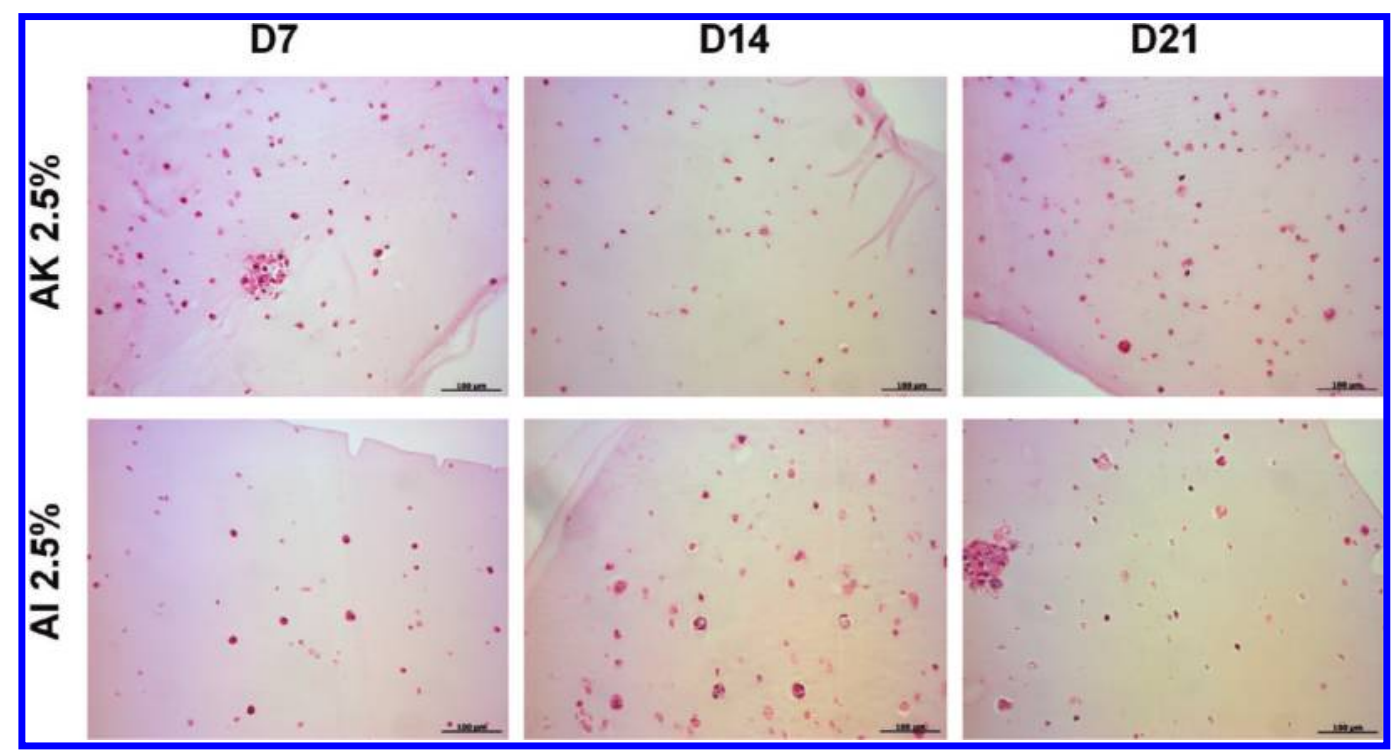

Figure 6. Haematoxylin eosin staining of ATDC5 chondrocytes encapsulated in alginate/ $\kappa$-carrageenan and alginate/ $l$-carrageenan type. The ATDC5 chondrocytes exhibited vacuoles morphology and cell clusters in $\kappa$-carrageenan formulation during the experiment, scale bars have $100 \mu \mathrm{m}$, and images are taken at $20 \times$ magnification.

$45 \%$ of their weight by the end of the study. Similar degradation behavior was found, for example, for hyaluronic acid hydrogel. ${ }^{34}$ The $2.5 \%$ alginate- $\kappa$-carrageenan hydrogels showed a slower degradation rate in DMEM medium than hydrogels exposed to PBS solution at $37{ }^{\circ} \mathrm{C}$ (Figure 3). The divalent cations used to keep hydrogels cross-linked will be exchanged by monovalent ones, leading to the formation of less crosslinked networks, as described elsewhere. ${ }^{35}$ This might be 
explained further by the higher content of ions in DMEM, which interact with the hydrogels of ionic nature and also related to the possible interaction of $\kappa$-carrageenan with the proteins presented in the DMEM medium composition. ${ }^{36}$ Concerning the mechanical properties it was found that the mechanical strength of the blends system (alginate/ $\kappa$ carrageenan) presented values around $0.7 \pm 0.25 \mathrm{MPa}$. Interestingly, the mechanical properties exhibited by the hydrogels in the wet state fall within the normal ranges of those reported for cartilage native tissue ${ }^{37}$ and are higher than the typical values presented for hydrogels like alginate, ${ }^{38}$ chitosan, ${ }^{39,40}$ and hyaluronic acid. ${ }^{41}$ The tensile strength of gels made from alginates reported in the literature appeared to be around $150-240$ and $250-280 \mathrm{kPa}^{42}$ These results clearly suggest that the compressive modulus of the developed hydrogels was improved by adding $\kappa$-carrageenan to the blends. It has been reported that the decreased sulfate content of carrageenan increases the strength of the hydrogel, thus, $\kappa$ carrageenan, which has less-sulfated groups, is expected to have better properties than $l$-carrageenan. ${ }^{25}$ Another important aspect for assessing the potential of these hydrogels for regenerative medicine applications is to evaluate their biocompatibility and their ability to sustain viability and proliferation of encapsulated cells. Because the microstructure and high water content are very similar to that of the extracellular matrix of natural cartilage, hydrogels are expected to preserve the viability of the cells. All hydrogels produced showed a noncytotoxic behavior. Nevertheless, lower polymeric concentrations and, consequently, less cross-linked networks, led to lower cell viability results, as a consequence of easier components release to the extraction medium. With increasing polymeric concentration the viscosity of the hydrogel also increases making it more stable when compared with the formulations with lower concentration, which lose their stability more easily due to the lower viscosity and cross-linking. Light and live cell imaging provided a wealth of information about the biophysics and biochemistry of cells encapsulated in the hydrogels. Figure 4 presents ATDC5 cells encapsulated in beads and fibers observed under light and fluorescence microscopy. Results from samples collected after 3 weeks of culture showed the presence of viable cells in both hydrogels formats as indicated by the positive staining with calcein-AM fluorescent dye. Three formulations (5:5, 7:3, and 8:2) corresponding to the two types of carrageenan, iota and kappa, in the blend with alginate were considered for the cell encapsulation assay. The results showed that the different ratio of alginate-carrageenan in the blend did not affect significantly the viability of the encapsulated ATDC5 chondrocytes. From the data presented in Figure 5A, we can easily observe higher viability for the formulations with the $\kappa$-carrageenan type compared with the metabolic activity register for the blends made with $l$-type. This cell behavior could be related to the higher sulfate composition of the $l$-carrageenan type. ${ }^{43}$ The influence of $\mathrm{CaCl}_{2} \cdot \mathrm{KCl}$ different concentration over the metabolic activity and proliferation of ATDC5 encapsulated cells showed that low concentration have higher cell viability (Figure 5B). The DNA quantification results complemented the data obtain from the MTS test (Figure 5C). After evaluating the influence of the polymeric concentration and the influence of the cross-linker over ATDC5 chondrocytes viability, it results clear that the first parameter is more important compared to the second. HE staining presented ATDC5 chondrocytes encapsulated within the hydrogel with typical cartilage spherical morphology (Figure 6), predicting a potential application of the hydrogel as an injectable scaffold in cartilage tissue engineering. These results further confirmed the biocompatibility of the developed hydrogels for cell encapsulation and delivery applications. Evaluation of these properties contributed to a further understanding of the formation mechanism of hydrogels and the biological applicability of these systems. As this process of the blend hydrogel formation is simple, feasible, and usually performed under mild conditions without employing any extraneous toxic cross-linking agents, we believe that such a matrix will have potential applications as cell delivery systems, wound management, drug delivery, cartilage tissue engineering, and other related biomedical fields.

\section{CONCLUSIONS}

We report herein the development of alginate-carrageenan hydrogels by a mild, friendly technique, yielding on using these carriers as cell delivery systems. The morphology, degradation, and mechanical behavior showed highly tunable properties without affecting their biocompatibility. Finally, the cell encapsulation assays revealed that alginate/ $\kappa$-carrageenan hydrogels presented a positive cellular response, supporting the viability and proliferation during long-term cell culturing. The thermo-gelling properties of such materials may also be used in new injectable systems that could be used to deliver cells through minimally invasive procedures. In fact, all the results obtain demonstrated that alginate/ $\kappa$-carrageenan can be an innovative and adequate alternative for the development of carrier systems to encapsulate cells or other bioactive agents of relevance in tissue engineering applications.

\section{AUTHOR INFORMATION}

\section{Corresponding Author}

*Tel.: +351 253510 906. Fax: +351 253510 909. E-mail: megomes@dep.uminho.pt.

\section{ACKNOWLEDGMENTS}

E.G.P. would like to acknowledge the Portuguese Foundation for Science and Technology for the Ph.D. grant (SFRH/BD/ 64070/2009). This work was partially supported by the European NoE EXPERTISSUES (NMP3-CT-2004-500283).

\section{REFERENCES}

(1) Baruch, L.; Machluf, M. Biopolymers 2006, 82, 570-9.

(2) Rinaudo, M. Polym. Int. 2008, 57, 397-430.

(3) d'Ayala, G. G.; Malinconico, M.; Laurienzo, P. Molecules 2008, 13, 2069-106.

(4) Drury, J. L.; Mooney, D. J. Biomaterials 2003, 24, 4337-51.

(5) Peppas, N.; Hilt, J.; Khademhosseini, A.; Langer, R. Adv. Mater. 2006, 18, 1345-1360.

(6) Sipahigil, O.; Dortunc, B. Int. J. Pharm. 2001, 228, 119-128.

(7) Stephen, A. M.; Phillips, G. O.; Williams, P. A. Food Polysaccharides and their Applications, 2nd ed.; CRC Press, Taylor \& Francis Group: New York, 2006; pp 205-244.

(8) Bhattacharyya, S.; Liu, H.; Zhang, Z.; Jam, M.; Dudeja, P. K.; Michel, G.; Linhardt, R. J.; Tobacman, J. K. J. Nutr. Biochem. 2009, 21, 906-913.

(9) Coviello, T.; Matricardi, P.; Marianecci, C.; Alhaique, F. J. Controlled Release 2007, 119, 5-24.

(10) Williams, P. Struct. Chem. 2009, 20, 299-308.

(11) Hennink, W. E.; van Nostrum, C. F. Adv. Drug Delivery Rev. 2002, 54, 13-36. 
(12) Ulijn, R. V.; Bibi, N.; Jayawarna, V.; Thornton, P. D.; Todd, S. J.; Mart, R. J.; Smith, A. M.; Gough, J. E. Mater. Today 2007, 10, 4048.

(13) Murua, A.; Portero, A.; Orive, G.; Hernandez, R. M.; de Castro, M.; Pedraz, J. L. J. Controlled Release 2008, 132, 76-83.

(14) Coradin, T.; Nassif, N.; Livage, J. Appl. Microbiol. Biotechnol. 2003, 61, 429-34.

(15) Wang, W.; Liu, X.; Xie, Y.; Zhang, H. a.; Yu, W.; Xiong, Y.; Xie, W.; Ma, X. J. Mater. Chem. 2006, 16, 3252-3267.

(16) Mammarella, E. J.; Rubiolo, A. C. Chem. Eng. J. 2003, 94, 7377.

(17) Gaserod, O.; Smidsrod, O.; Skjak-Braek, G. Biomaterials 1998, $19,1815-25$.

(18) Gaserod, O.; Sannes, A.; Skjak-Braek, G. Biomaterials 1999, 20, $773-83$.

(19) Poncelet, D.; Neufeld, R. J.; Goosen, M. F. A.; Burgarski, B.; Babak, V. AlChE J. 1999, 45, 2018-2023.

(20) Mohamadnia, Z.; Zohuriaan-Mehr, M. J.; Kabiri, K.; Jamshidi, A.; Mobedi, H. J. Biomater. Sci., Polym. Ed. 2008, 19, 47-59.

(21) Correlo, V. M.; Boesel, L. F.; Pinho, E.; Costa-Pinto, A. R.; Alves da Silva, M. L.; Bhattacharya, M.; Mano, J. F.; Neves, N. M.; Reis, R. L. J. Biomed. Mater. Res., Part A 2009, 91, 489-504.

(22) Salgado, A. J.; Coutinho, O. P.; Reis, R. L. Tissue Eng. 2004, 10, 465-74.

(23) Gomes, M. E.; Reis, R. L.; Cunha, A. M.; Blitterswijk, C. A.; de Bruijn, J. D. Biomaterials 2001, 22, 1911-7.

(24) Atsumi, T.; Miwa, Y.; Kimata, K.; Ikawa, Y. Cell Differ. Dev. 1990, 30, 109-16.

(25) Distantina, S.; Wiratni.; Fahrurrozi, M.; Rochmadi. In Carrageenan Properties Extracted From Eucheuma cottonii, Indonesia; World Academy of Science, Engineering and Technology: New Mexico, USA, 2011; pp 738-742.

(26) Spiller, K. L.; Maher, S. A.; Lowman, A. M. Tissue Eng., Part B 2011, 17, 281-99.

(27) Lin, C. C.; Metters, A. T. Adv. Drug Delivery Rev. 2006, 58, 1379-408.

(28) Langer, R.; Peppas, N. A. AlChE J. 2003, 49, 2990-3006.

(29) Kuo, C. K.; Ma, P. X. Biomaterials 2001, 22, 511-521.

(30) Xu, J. B.; Bartley, J. P.; Johnson, R. A. J. Membr. Sci. 2003, 218, $131-146$.

(31) Lim, Y.-M.; Gwon, H.-J.; Choi, J.-H.; Shin, J.; Nho, Y.-C.; Jeong, S.; Chong, M.; Lee, Y.-M.; Kwon, I.; Kim, S. Macromol. Res. 2010, 18, 29-34.

(32) Roh, Y. H.; Shin, C. S. J. Appl. Polym. Sci. 2006, 99, 3483-3490.

(33) Kachouie, N. N.; Du, Y. A.; Bae, H.; Khabiry, M.; Ahari, A. F.; Zamanian, B.; Fukuda, J.; Khademhosseini, A. Organogenesis 2010, 6, 234-244.

(34) Ito, T.; Yeo, Y.; Highley, C. B.; Bellas, E.; Benitez, C. A.; Kohane, D. S. Biomaterials 2007, 28, 975-83.

(35) Kuo, C. K.; Ma, P. X. J. Biomed. Mater. Res., Part A 2008, 84, 899-907.

(36) Arltoft, D.; Ipsen, R.; Madsen, F.; de Vries, J. Biomacromolecules 2007, 8, 729-736.

(37) Fisher, J. P.; Jo, S.; Mikos, A. G.; Reddi, A. H. J. Biomed. Mater. Res., Part A 2004, 71, 268-74.

(38) Knight, M. M.; Bravenboer, J. V. D. B.; Lee, D. A.; van Osch, G. J. V. M.; Weinans, H.; Bader, D. L. Biochim. Biophys. Acta, Gen. Subj. 2002, 1570, 1-8.

(39) Martins, A. M.; Santos, M. I.; Azevedo, H. S.; Malafaya, P. B.; Reis, R. L. Acta Biomater. 2008, 4, 1637-1645.

(40) Murakami, R.; Takashima, R. Food Hydrocolloids 2003, 17, 885-888.

(41) Burdick, J. A.; Prestwich, G. D. Adv. Mater. 2011, 23, H41H56.

(42) Wang, L.; Shelton, R. M.; Cooper, P. R.; Lawson, M.; Triffitt, J. T.; Barralet, J. E. Biomaterials 2003, 24, 3475-81.

(43) Luna, S. M.; Gomes, M. E.; Mano, J. F.; Reis, R. L. J. Bioact. Compat. Polym. 2010, 25, 341-359. 